\title{
Pragmaticalización y lenguas clásicas: el caso de latín abi
}

\author{
Luis Unceta Gómez
}

\section{Pragmaticalization and Classical languages: the case of Latin abi}

\begin{abstract}
This paper aims at offering an analysis of the pragmatic functions developed by the Latin particle $a b i$, originally the imperative of the verb abeo ('to leave', 'to go away'): discourse marker of conversation closing, directive pragmatic marker, expression of disagreement and expression of congratulation. Based specially on the evidences of Roman Comedy, a proposal of pragmatic evolution is offered, in agreement with similar pragmaticalization paths in other languages.
\end{abstract}

\section{Keywords}

abi; Roman comedy; discourse and pragmatic marker; pragmaticalization; subjectification 


\section{Introducción}

Desde un punto de vista morfológico, la forma latina abi es el imperativo de presente del verbo abire, 'salir, irse, marcharse'. ${ }^{1}$ Sin embargo, más allá de ese valor, esta unidad experimentó en época temprana una especialización en funciones comunicativas bien reconocibles en los textos cómicos y aparentemente contradictorias entre sí. La bibliografía previa le ha atribuido valores como la orden, el rechazo irritado o la aprobación, ${ }^{2}$ pero ni explican las relaciones entre ellos, ni su posible origen. Apoyándose en estas funciones comunicativas, la descripción gramatical tradicional ha querido ver en abi una interjección secundaria (Löfstedt 1966: pp. 96-97; Hofmann ${ }^{32003: ~ p . ~ 151), ~ c o n s i d e r a-~}$ ción que ha afectado a otros imperativos gramaticalizados (como puta, uide, mane, caue, tene, age o em, ${ }^{3}$ etc.), algo que ha generado la falsa impresión de que la lengua latina posee un número elevado de interjecciones impropias (Unceta Gómez 2016a). Sin embargo, a pesar de las dificultades que conlleva (cf. Jacobs \& Jucker 1995), si asumimos una perspectiva pragmática para el análisis de las lenguas clásicas y partimos de una definición restrictiva y no conceptualista de las interjecciones (Unceta Gómez 2012), resulta enseguida evidente que la mayor parte de esos imperativos gramaticalizados no pueden considerarse integrantes de esa categoría, puesto que sus funciones discursivas son diferentes de las que podemos considerar propiamente interjectivas.

Partiendo de estas premisas, en este trabajo trataré de determinar las funciones comunicativas reconocibles en abi y las líneas evolutivas que han propiciado esos valores. Para ello, me serviré de dos conceptos fundamentales. El primero es el de pragmaticalización, que aborda el desarrollo de funciones pragmáticas en lexemas de distintas categorías. En la consideración de este fenómeno, me adhiero a la opinión de quienes piensan que la pragmaticalización es un subtipo de gramaticalización, entendida esta última como un fenómeno amplio de cambio lingüístico (Dostie 2004; Company Company 2004; Diewald 2011; Ghezzi 2014). La decategorización, la pérdida de propiedades sintácticas o el debilitamiento del significado conceptual en favor del procedimental son rasgos frecuentemente invocados como evidencia de este tipo de cambio, que da lugar a marcadores del discurso y marcadores pragmáticos. ${ }^{4}$

1 «El preverbio $a b$ - indica "alejamiento de un límite simple" $(\mid \rightarrow)$, en oposición a ad- que expresa "aproximación a un límite simple" $(\rightarrow \mid)$. [...] Abire ("irse") es más preciso que el simple ire ("ir") en la indicación del punto de partida» (García Hernández 2015: p. 112).

2 Hofmann (32003: p. 151) le dedica uno de los análisis más completos en su obra sobre el latín coloquial. El Oxford Latin Dictionary no lo incluye como entrada independiente y se limita a señalar (s.u. abeo, epígrafe 6 , p. 5, col. 1), tras la indicación «coloquial», los siguientes valores: A. abi in malam rem (cum): «go to the devil, to hell (with you and your -)!». B. abi hinc cum: «hence with your - ! away with you! enough!». C. (w. expressions of aproval) «good for you!». De manera semejante, el Thesaurus Linguae Latinae incluye los valores de abi en la entrada correspondiente a abeo y califica (cols. 66-67) los usos de abi como formulae in comoedia tritae, organizándolos como sigue: A. cum deuotione. B. cum improbatione. C. cum laude.

3 Cf., entre otros, Barbini (1966), trabajo de elocuente título. Unceta Gómez (2017) ofrece un acercamiento pragmático a $\mathrm{em}$.

4 Sigo aquí la propuesta terminológica de Ghezzi (2014: p. 15), quien distingue entre dos categorías de marcadores funcionales: marcadores del discurso («elements that index the structure of discourse, fun- 
El segundo es el concepto de (inter)subjetivación; tal como lo plantea Traugott en su formulación más clásica $(1989,1995)$, este proceso de evolución semántica se sustancia en tres tendencias:

Tendency I: $\quad$ Meanings based in the external described situation > meanings based in the internal (evaluative/perceptual/cognitive) described situation.

Tendency II: Meanings based in the external or internal described situation > meanings based in the textual and metalinguistic situation.

Tendency III: Meanings tend to become increasingly based in the speaker's subjective belief state/attitude toward the proposition. (Traugott 1989: pp. 34-35)

Aunque esta investigación ha tenido en cuenta el conjunto de textos latinos de la Antigüedad (hasta $c a .200$ d.C.), ${ }^{5}$ el grueso de los testimonios de abi se concentra en el latín arcaico y, en concreto, en las comedias Plauto y Terencio (cf. Tabla 1). Este hecho se justifica, al menos en parte, por dos factores: la pertenencia de abi al registro coloquial $^{6}$ (para el que este género es una de nuestras principales fuentes de información) y la idoneidad de los textos dialógicos, especialmente aquellos que implican la presencia del interlocutor, para la observación de los valores pragmáticos de unidades como esta.

\begin{tabular}{|l|c|c|}
\hline \multicolumn{1}{|c|}{ Autor Época } & 134 \\
\hline Plauto & $c a .254-184$ a.C. & 2 \\
\hline Cecilio Estacio & $c a .220-c a .166$ a.C. & 38 \\
\hline Terencio & $c a .195 / 185-c a .159$ a.C. & 1 \\
\hline Afranio & $c a .154$ a.C.- '? & 1 \\
\hline Cicerón & $106-43$ a.C. & 1 \\
\hline Virgilio & $70-19$ a.C. & 3 \\
\hline Horacio & $65-8$ a.C. & 9 \\
\hline Tito Livio & 59 a.C. -17 d.C. & 4 \\
\hline Ovidio & 43 a.C. -17 d.C. & 2 \\
\hline Quintiliano & $c a .35-c a .95$ d.C. & 1 \\
\hline Valerio Máximo & s. I d.C. & 1 \\
\hline Curcio Rufo & s. I d.C. & 7 \\
\hline Estacio & $c a .45-96$ d.C. & 2 \\
\hline Apuleyo & $c a .124-c a .170$ d.C. & 1 \\
\hline Juvenal & s. I-s. II d.C. & \\
\hline
\end{tabular}

Tabla 1. Distribución diacrónica de abi.

Este trabajo se organiza del siguiente modo: tras analizar los casos en los que es perceptible en mayor o menor grado el contenido etimológico de abi (Sección 2), se abordan los usos de esta unidad como marcador discursivo, en su función de cierre conversacional

ctioning as discourse-cohesive devices that stress intra-discourse relations») y marcadores pragmáticos («items that have an (inter)subjective meaning»).

5 El corpus analizado en este trabajo se basa en los datos que presenta el ThLL cotejados con las correspondientes ediciones.

6 Para una puesta al día del concepto de latín coloquial, véase Chahoud (2010). 
(Sección 3.1), y como marcador pragmático, categoría en la que abi desarrolla funciones de refuerzo del acto de habla directivo (Sección 3.2), de marca de desacuerdo y reproche (Sección 3.3) y de aprobación y felicitación (Sección 3.4). Finalmente, la Sección 4 presenta las conclusiones del estudio y ofrece una propuesta de interpretación del proceso evolutivo de todos estos valores.

\section{Contenido etimológico y su persistencia}

El sentido propio del imperativo abi es perceptible en la mayor parte de sus usos (véase infra, Tabla 2). Es muy claro, por ejemplo, en los casos en los que el interlocutor explicita su voluntad a la obediencia de la orden a través de los mismos lexemas, como en (1): ${ }^{7}$

(1) EVC. Tace atque abi intro. STA. Taceo atque abeo. EVC. Occlude sis (Plaut., Aul. 103). ['EUCLIÓN. Cállate y entra. ESTÁFILA. Me callo y entro. EUCLIÓN. Haz el favor de cerrar la puerta.']

Prueba también del mantenimiento de su significado léxico y su función directiva como imperativo es la posibilidad de ser negado, como en (2) o de recibir alguna forma de intensificación o de mitigación, como en (3) y (4):

(2) PHR. Mane, aliquid fiet: $\boldsymbol{n e}$ abi. ${ }^{8}$ [...] (Plaut., Truc. 366).

['Espera. Encontraremos una solución. No te vayas.']

(3) TH. [...] Age abi, abi impune. Em huic habeto gratiam (Plaut., Most. 1180). ${ }^{9}$

['Anda, vete, vete: te perdono. A él es a quien has de agradecérselo.']

(4) TR. [...] Abi quaeso hinc domum (Plaut., Most. 578). ${ }^{10}$

['Vete, por favor, a casa.']

Pero el valor de movimiento del lexema base resulta particularmente nítido en aquellos usos en los que se detalla el modo de movimiento, como en (5) y (6), o el lugar al que interlocutor debe dirigirse. En ocasiones, esta última especificación resulta relevante o incluso imprescindible para el correcto cumplimiento de una determinada instrucción. Así se aprecia en (7):

(5) PL. Abi sane ad litus curriculo, Trachalio (Plaut., Rud. 855).

['Anda, Tracalión, vete de una carrera a la playa.']

7 Los textos latinos de Plauto y Terencio proceden de las ediciones oxonienses de W. M. Lindsay, y R. Kauer \& W. M. Lindsay, respectivamente. Las traducciones de los cómicos están basadas en las de J. Román Bravo, con algunas modificaciones (Plauto - Terencio, Comedia latina. Obras completas de Plauto y Terencio. Traducción de J. Román Bravo. Edición, introducciones y notas de R. López Gregoris, Madrid, Cátedra, Bibliotheca Aurea, 2012). Salvo indicación contraria, las traducciones de los textos del resto de autores son propias.

8 Negación del imperativo que tradicionalmente se ha entendido como propia del latín hablado y con ciertos vestigios en las lenguas romances (cf. Löfstedt 1966: pp. 63-66), cuya frecuencia de aparición es muy minoritaria con respecto a las formas de subjuntivo negado (ne abeas), con el que se constituyó un paradigma bien asentado.

9 Véase también Plaut., Most. 584 o Persa 195, donde aparece intensificado con modo. En el ejemplo (5) se combina igualmente con sane. Sobre el papel de estas unidades en oraciones directivas, cf. Risselada (1994).

10 Cf. Plaut., Cas. 214 (amabo). 
(6) Abi ad thensaurum iam confestim clanculum (Plaut., Trin. 798).

['Vete inmediatamente junto al tesoro y procura que no te vean.']

(7) Nunc tu abi ad forum ad erum et narra haec ut nos acturi sumus (Plaut., Asin. 367).

['Ahora tú vete al foro a ver al amo y cuéntale cómo vamos a actuar.']

En las comedias (y especialmente en las plautinas) resultan muy frecuentes otras especificaciones genéricas; estas son, en orden de uso creciente, rus (8), domum (9) o intro (10): ${ }^{11}$

(8) TH. Nunc abi rus, dic me aduenisse filio. TR. Faciam ut iubes (Plaut., Most. 929). ['TEOPRÓPIDES. Ahora vete al campo y dile a mi hijo que he llegado. TRANIÓN. Cumpliré tus órdenes.']

(9) Abi domum, iube uasa pura actutum adornari mihi (Plaut., Amph. 1126).

['Vete a casa y hazme preparar los vasos sagrados.']

(10) LY. Intro abi, uxor, atque adorna nuptias. CL. Faciam ut iubes (Plaut., Cas. 419).

['LISIDAMO. Entra dentro, mujer, y prepara la boda. CLEÓSTRATA. Haré como ordenas.']

En buena parte de los casos en que aparecen las dos últimas determinaciones (sobre todo la última), se reconoce claramente en las comedias una función metateatral, de especificación del movimiento escénico, en la que este verbo anticipa en cierto modo la salida de escena de un determinado personaje. ${ }^{12}$ Con este valor, nos encontramos ya cerca de la primera función que desarrolla abi como marcador discursivo, en la regulación del intercambio comunicativo (infra, § 3.1).

Con todo, la especificación del destino no siempre es necesaria y, en ocasiones, el verbo de movimiento proporciona una información irrelevante por evidente. De tal manera, en un número de casos amplio, aunque el sentido propio del verbo sea aún reconocible, la combinación sintagmática del imperativo de un verbo de movimiento y (con o sin conjunción copulativa) un segundo imperativo que explicita léxicamente el contenido del acto directivo (i/uade/age [et/ac/atque] fac) constituye más bien una construcción idiomática muy fijada en latín y con paralelos en otras muchas lenguas, tanto antiguas como modernas. ${ }^{13}$ Löfstedt (1966: pp. 82-90) denomina esta estructura «imperativo asociativo». Además de en los ejemplos (7)-(10), es posible reconocer esta

11 Además del acusativo de dirección o la especificación adverbial, se documenta también el uso del acusativo interno: abi tacitus tuam uiam (Plaut., Rud. 1027); véase García Hernández (2015: p. 113). Por el contrario, resulta muy inusual la especificación del origen (como en el ejemplo de (4)), puesto que, al tratarse del imperativo de un verbo de movimiento orientado, abire exige un anclaje local muy determinado: el de la interacción comunicativa (sobre el complemento en ablativo como marca de origen, puede verse Torrego 2009: p. 236, y Pinkster 2015: pp. 125-126). Tal vez la escasa relevancia pragmática de este complemento (por su fácil restitución contextual) sea, como señala uno de los evaluadores anónimos de este trabajo, uno de los motivos que expliquen el 'vaciado' semántico de abi.

12 Véase González Vázquez (2014: s.u. abeo), quien señala que «[e]l movimiento escénico tiene un sistema fijo y recurrente. La referencia espacial es el escenario y la "trasescena", sobre los que se mueven los actores desde el punto de vista del personaje, no del espectador. El movimiento escénico se divide en: salida a escena, salida de escena y movimiento por la escena».

13 En latin, el verbo más frecuente en esta construcción es ire y sus modificados, construcción que pervive a lo largo de toda la latinidad (Löfstedt 1966: p. 83), si bien, a partir de la Biblia $i$ es muy frecuentemente sustituido por uade (ibid.: pp. 87-88). Mauri \& Sansò (2014) ofrecen una muestra amplia de este tipo de construcciones en diferentes lenguas de todo el mundo. 
construcción en los siguientes; en (11) encontramos ambas indicaciones yuxtapuestas, mientras que (12) y (13) incluyen la conjunción copulativa et:

(11) [...] Abi, deprome. [...] (Plaut., Curc. 255).

['Ve y saca las cosas (de la despensa).']

(12) PI. Non it. †negato esse† ituram. Abi et renuntia (Plaut., Bacch. 592).

['No se va; dice que no se irá. Ve y comunícale la respuesta.']

(13) Tu illas abi et transduce. [...] (Ter., Ad. 917). ${ }^{14}$

['Tú vete a buscarlas.']

Aunque en la mayoría de estos usos se reconozca aún en abi la persistencia de su contenido etimológico de desplazamiento, ${ }^{15}$ la automatización de esta construcción desembocará en la pragmaticalización de esta unidad, cuya naturaleza imperativa dará lugar a un valor intensificador del acto de habla directivo (infra, § 3.2).

\section{Abi como marcador discursivo y pragmático}

\subsection{Regulador de la conversación}

La primera función en la que es posible reconocer en abi un valor discursivo derivado del significado léxico del verbo abire en imperativo es la regulación de la conversación. ${ }^{16}$ En concreto, se observa en abi una función iniciativa de cierre, ${ }^{17}$ que señala sencillamente el cumplimiento de los objetivos conversacionales. Si la interacción se produce en sentido jerárquico descendente, es decir, es el amo el que da instrucciones a un siervo, no se tiene en cuenta la amenaza que hacia la imagen negativa del interlocutor puede suponer un cierre de estas características. ${ }^{18}$ Así se aprecia en (1) y (14), donde el viejo

14 Sobre el orden de palabras, cf. Löfstedt (1966: p. 83), quien señala: «le verbe associatif et le verbe principal font un groupe fixe devant lequel se placent les compléments d'objet». Esta fijación sintagmática puede considerarse indicio de la gramaticalización de esta construcción.

15 La persistencia del contenido semántico originario de las unidades léxicas es un fenómeno muy frecuente en los procesos de gramaticalización y pragmaticalización, que dificulta en muchas ocasiones la determinación de la naturaleza exacta de una pieza léxica determinada.

16 Como señala Roesch (2002: p. 318), la ruptura de diálogo más frecuente se manifiesta en la separación física de los personajes sobre la escena. «On le voit à travers l'emploi omniprésent du verbe $e o$, et surtout du préverbé abeo, en fin de dialogue. Soit le personnage annonce lui-même qu'il va partir, et, en cela, clôt la discussion, soit il part en obéissant à l'injonction abi, qui lui a été faite par un autre personnage». Una de las funciones esenciales de estos elementos del diálogo teatral es la de codificar los movimientos de los personajes, dada la ausencia de acotaciones o didascalias que las precisen.

17 En español, el marcador pragmático venga puede asumir también función de cierre de turno, aunque, por lo general, constituye un acto preparatorio y da al interlocutor la posibilidad de continuar con la conversación; cf. Blas Arroyo (1998: pp. 558-562).

18 Sobre el análisis de la cortesía lingüística en latín, puede verse Unceta Gómez (2014). El esclavo, por el contrario, emplea fórmulas iniciativas de cierre deferentes del tipo nisi quid uis (Roesch 2002: pp. 324-325) o numquid (me) uis?/numquid aliud? (Hough 1945; Roesch 2002: pp. 327-330), que dejan al amo la responsabilidad de finalizar el intercambio comunicativo. Así, por ejemplo, en PA. Eo nunciam, / nisi quippiam / remorare me. / LY. Abi et cura (Plaut., Cas. 715-718), donde abi puede considerarse reactivo. Véase también Plaut., Mil. 574-575. 
Hegión tras dar indicaciones a su esclavo lorario (encargado de aplicar los castigos físicos a sus compañeros de esclavitud), lo despide así:

(14) Sed sati' uerborumst. Cura quae iussi atque abi (Plaut., Capt. 125).

['Pero, basta ya de conversación. Encárgate de lo que te he ordenado y vete.']

Sin embargo, si entre los interlocutores no existe una relación jerárquica tan marcada, el empleo de esta unidad puede resultar excesivamente abrupto, si no va acompañado de un acto preparatorio y se prescinde de otras fórmulas especializadas en la despedida. Se observa en (16), donde la proxeneta Cleéreta corta la conversación con el joven Diábolo (que quiere contratar los servicios de una de sus chicas), aunque este se muestra renuente a ello: ${ }^{19}$

(15) CL. Remeato audacter, mercedem si eris nactus: nunc abi.

DIA. Mane, mane, audi. Dic, quid me aequom censes pro illa tibi dare, annum hunc ne cum quiquam alio sit? CLE. Tene? Viginti minas;

atque ea lege: si alius ad me prius attulerit, tu uale.

DIA. At ego est etiam priu' quam abis quod uolo loqui. CL. Dic quod lubet (Plaut., Asin. 228-232).

['CLEÉRETA. Cuando consigas con qué pagar, vuelve sin miedo; ahora vete. DIÁBOLO. iEspera, espera, escucha! Dime: ¿cuánto consideras justo que te dé por ella, para que en todo el año no esté con ningún otro? CLÉERETA. ¿Tú? Veinte minas; y con esta condición: si otro me las trae antes, adiós muy buenas. DIÁBOLO. Espera, todavía hay otra cosa que quiero decirte antes de que te vayas. CLEÉRETA. Di lo que quieras.']

En cambio, en el siguiente diálogo entre esclavos, $a b i$ es utilizado por Pegnio para iniciar el cierre de una conversación que no está siendo colaborativa por parte de su interlocutora, Sofoclidisca, pero no impide la aparición de una fórmula de despedida menos brusca, como es ualeas:

(16) PA. Par pari respondes dicto. Abi iam, quando ita certa rest.

Nihili facio scire. Valeas. SO. Asta [...] (Plaut., Persa 223-224).

['PEGNIO. Veo que respondes a mis preguntas con idénticas preguntas. Vete de una vez, si así lo quieres. No tengo interés en saberlo. Adiós. SOFOCLIDISCA. Espera.']

\subsection{Refuerzo del acto de habla directivo}

Como se ha mencionado (supra, § 2), se reconocen plasmaciones de la combinación sintagmática «abi + imperativo» en las que la idea de movimiento es aún perceptible. A excepción de los casos en los que abi va acompañado de la especificación del lugar, en muchas ocasiones la indicación del desplazamiento, aunque pueda resultar pertinente, es innecesaria. Así ocurre, por ejemplo, en (11), donde la acción de «sacar» exige un desplazamiento previo a un lugar interior. En estos casos, como señala Löfstedt (1966:

19 Véase además Plaut., Cist. 117-119, en una conversación que remeda hábitos lingüísticos que podemos considerar corteses. 
p. 84), es posible que el verbo de movimiento antes de la orden principal sirva para subrayar una idea de rapidez e inmediatez necesarias para el cumplimiento de esa indicación. Sea como sea, en el valor semántico de $a b i$ se aprecia frecuentemente el desvanecimiento de la especificación local que aporta el preverbio.

En efecto, la automatización de la combinación sintagmática denominada por Löfstedt «imperativo asociativo», como se ha adelantado, da lugar a la pérdida o vaciamiento de rasgos semánticos («bleaching») de $a b i$, que pasa a utilizarse como mera intensificación del acto de habla directivo, combinándose incluso con imperativos cuya ejecución no requiere un desplazamiento previo..$^{20}$ Como se aprecia en (17), el movimiento de salida es irrelevante, por estar incluido en los verbos remouere o auferre, o, en todo caso, debería realizarse en un orden distinto al planteado:

(17) 'Remoue, abi aufer': neminem eorum haec adseuerare audias (Plaut., Mil. 761).

[“'Retíralo, anda llévatelo": a ninguno de ellos oirás decir tales cosas.']

Del mismo modo, en los ejemplos de (18) y (19), no se espera un movimiento físico para la ejecución del acto directivo principal: en (18) porque en realidad no se solicita que se abandone la escena, sino una idea; en (19) porque es precisamente Cleóstrata quien tiene intención de marcharse (como se aprecia en la pregunta que inmediatamente le dirige su marido, Lisidamo, quo nunc abis? [v. 231])::21

(18) ANTE. Ausculta sorori. Abi, diiunge inimicitias cum inprobo.

HA. Hoc age sis, leno. Quamquam ego te meruisse ut pereas scio, non experiar tecum. [...] (Plaut., Poen. 1406-1408).

['ANTERÁSTILE. Haz caso a mi hermana. Va, renuncia a pelearte con un canalla. HANÓN. Venga, escúchame, lenón. Aunque estoy convencido de que mereces la muerte, no me querellaré contigo.']

(19) [LY.] Vxor mea meaque amoenitas, quid tu agis? CL. Abi atque abstine manum (Plaut., Cas. 229).

['LISIDAMO. Esposa mía, cariño, ¿̇cómo te encuentras? CLEÓSTRATA. Quita y aparta esa mano.']

La culminación de esta evolución se observa en el ejemplo (20), donde la marcha del interlocutor es incompatible con la ejecución de la acción principal, lo que certifica el reanálisis de esta unidad:

(20) PH. Abi, tange: si non totu' friget, me enica (Ter., Phorm. 994).

['Anda, tócalo: si no está helado de pies a cabeza, mátame.']

20 El fenómeno, muy frecuente en lenguas de todo el mundo, es definido por Mauri \& Sansò (2014: pp. 175-176) en los siguientes términos: «in directive speech acts the addressee often needs to move away from the place where the speaker utters his/her order as a preliminary action necessary to bring about the desired SoA [state of affairs]. In most cases, the directive strategies employed by the speaker leave this need for dislocation unexpressed and implicit: when it is explicit, however, i.e. when a sequence of two verbs is used ( go and do $x /$ go do $x$ ), the verb expressing dislocation can be easily reinterpreted as a general directive marker. In other words, the frequency with which in directive situations the realization of the order implies a dislocation of the performer may be speculatively considered as a prerequisite for the construction [go do $\mathrm{X}$ ] to be processed as a single unit and, subsequently, for the verb meaning 'go' to be reanalyzed as having a general (i.e. non-dislocative) directive function».

21 Como señala Löfstedt (1966: p. 84) esta evolución se constata también con el imperativo del verbo simple. Véase, por ejemplo, quaeris ubi inuenias: artes, i, perlege nostras (Ou., Rem. 487). 
En casos como este, $a b i$ se ha transformado en una partícula que, en combinación con un imperativo, enfatiza el acto de habla directivo, en una evolución muy semejante a la de la forma de imperativo age ${ }^{22}$ altamente pragmaticalizada ya en latín arcaico, o el marcador pragmático español venga..$^{23}$

\subsection{Desacuerdo y reproche}

Siendo aún perceptible el significado de desplazamiento del lexema base, existe también un significativo número de casos en los que se observa un uso metafórico de $a b i$, según el cual la especificación de lugar se refiere a un castigo o un hecho negativo, que delata la indignación del locutor. ${ }^{24}$ Además de algunas formulaciones más creativas, como (21), existen otras más fijadas - in malam rem (22), in malum cruciatum (23), in crucem (24) -, cuyo significado intencional, claramente conflictivo, es muy similar al de expresiones semejantes en español como «vete a paseo», «vete a la porra», o la disfemística «vete a la mierda»:

(21) AG. Potesne mi auscultare? MI. Possum. AG. Abi domum ac suspende te (Plaut., Poen. $309){ }^{25}$

['AGORASTOCLES. ¿Serías capaz de seguir un consejo mío? MILFIÓN. Por supuesto. AGORASTOCLES. Pues vete a casa y cuélgate.']

(22) HE. Abi in malam rem, ludis me. [...] (Plaut., Capt. 877).

['Vete al diablo. Te estás burlando de mí.']

(23) EVC. [...] Molestus ne sis! I cenam coque,

aut abi in malum cruciatum ab aedibus. CON. Abi tu modo (Plaut., Aul. 458-459).

['EUCLIÓN. iNo me des la lata! Vete a preparar la cena o lárgate de casa y vete a que te crucifiquen. CONGRIÓN. Vete tú.']

(24) SA. Abi intro - in crucem. [...] (Plaut., Persa 856).

['SAGARISTIÓN. Vete dentro a que te crucifiquen.']

A partir de este tipo de expresiones y sin necesidad de especificación adverbial, $a b i$ se especializa en el reproche o el rechazo enérgico e irritado de las palabras del interlocutor. Por lo general, se especifica el motivo exacto del reproche, como en (25), (26) y (27), ejemplo este último en el que el rechazo no se refiere a las palabras del interlocutor, sino a su actitud: ${ }^{26}$

22 Sobre el funcionamiento de age, véase Unceta Gómez (2009: pp. 62-63).

23 El Diccionario de partículas discursivas del español (Briz \& Pons \& Portolés 2008: s.u.) define este uso de venga en los siguiente términos: «Llama la atención del destinatario y le anima a actuar según lo que se va a decir o solicitar a continuación o lo que se ha dicho o solicitado anteriormente». Véase también Blas Arroyo (1998: pp. 551-552) y Daniels (2014: pp. 228-231).

24 Como señalan Sansò \& Mauri (2014: p. 177) en la evolución de verbo de movimiento a marcador pragmático en construcciones directivas, se constata con frecuencia la aparición de matices emocionales: «Overtones of anger and disapproval, correlated with the 'subjective' construal of distance between the speaker and the addressee, may also arise at this stage and take over the original motion meaning». Cf. Ter., And. 255.

26 Véase también Ter., Eun. 221 (abi, nil dicis); Plaut., Mil. 324 y Most. 1080 (abi ludis me); Plaut., Trin. 972 
(25) SC. Profecto uidi. PA. Tutin? SC. Egomet duobus his oculis meis.

PA. Abi, non ueri simile dicis neque uidisti. [...] (Plaut., Mil. 290-291).

['ESCELEDRO. Te juro que lo vi. PALESTRIÓN. ¿Tú mismo? ESCELEDRO. Yo mismo, con estos dos ojos míos. PALESTRIÓN. Venga ya. Eso no hay quien se lo crea y tú no los has visto.']

(26) SY. Numquam rem facies: abi, nescis inescare homines, Sannio (Ter., Ad. 220).

['Jamás harás fortuna. Vete a paseo. No sabes engatusar a la gente, Sanión.']

(27) LY. Vbi id est aurum? L. S. In arca apud me. Nunc uolo me emitti manu.

LY. Egone te emittam manu, scelerum cumulatissume?

L. S. Abi, ere, scio quam rem geras.

Lepide hercle animum tuom temptaui. Iam ut eriperes apparabas (Plaut., Aul. 823-827). ['LICÓNIDES. ¿Y dónde está ese oro? ESTRÓBILO. En un arca en mi habitación. Ahora quiero que me des la libertad. LICÓNIDES. ¿Que te dé la libertad, grandísimo bellaco? ESTRÓBILO. Venga ya, amo; ya veo tus intenciones. Fue una bonita forma de probarte, por Hércules. Ya estabas dispuesto a quitármelo.']

Por otra parte, son varias las ocasiones en que el carácter conflictivo de la situación se intensifica con la combinación con insultos, como se observa en (28), ${ }^{27}$ y puede ir acompañado de una amenaza (29):

(28) [...] HE. Abi, stultu's, sero post tempus uenis (Plaut., Capt. 870).

['Anda a paseo; eres un tonto. Llegas tarde, a destiempo.']

(29) TO. Inspicere morbum tuom lubet. SA. Ah, ah! ${ }^{28}$ Abi atque caue sis

a cornu. [...] (Plaut., Persa 316-317).

['TÓXILO. Me gustaría examinar tu mal. SAGARISTIÓN. iEh, eh! Aparta. Ten cuidado no vayas a recibir una cornada.']

El ejemplo (30) certifica el mantenimiento de esta función en la imitación de la lengua hablada que nos proporciona una obra de época posterior:

(30) Tunc illa capite quassanti: 'abi' inquit, 'fatue, qui in domo funesta cenas et partes requiris' (Apul., Met. 2, 24, 4).

['Ella entonces, moviendo la cabeza: "Vete a paseo, impertinente - me dice -. En las fúnebres circunstancias de esta casa, hablas de comer y reclamas tu parte”.'] ${ }^{29}$

(abi sis, nugator: nugari nutatori postulas). Un valor más benévolo tiene su uso en Plaut., Persa 490 (abi, ne iura, sati' credo).

27 Cf. además Plaut., Most. 569 (abi sis, belua); Ter., Phorm. 59 (abi sis, insciens). En estos ejemplos, así como en (29), el marcador pragmático sis, tradicionalmente entendido como un atenuador cortés («si quieres», «por favor»), tiene una función intensificadora, tal y como lo concibe Dickey (2006), quien le atribuye una función primaria de marcación de foco.

28 La interjección $a h$ ! expresa aquí una idea de fastidio (Unceta Gómez 2012: p. 387).

29 Traducción de L. Rubio Fernández (Madrid: Gredos 1978). 


\subsection{Aprobación y felicitación}

Existe, por último, una función de $a b i$ desligada por completo del contenido semántico del verbo del que deriva y aparentemente inconexa con respecto a las analizadas hasta aquí: la aprobación o la felicitación. Con este valor, la partícula puede combinarse con la explicitación léxica de este acto de habla, como se comprueba en (31), (32) y (33):

(31) DE. Laudo: Ctesipho, patrissas: abi, uirum te iudico (Ter., Ad. 564).

['Te felicito, Ctesifón, sales a tu padre. iVamos! Te considero un hombre.']

(32) Asta igitur, ut consuetus es puer olim. Scin ut dicam?

Em sic. Abi, laudo, nec te equo magis est equos ullus sapiens (Plaut., Asin. 704). ${ }^{30}$

['Ponte, pues, como solías hacerlo cuando eras niño. ¿Sabes cómo te digo? (ARGIRI-

PO se coloca.) Sí, así. iVamos! Te felicito. No hay otro caballo más inteligente que tú.']

(33) Abi, laudo, scis ordine ut aequomst tractare homines; [...] (Plaut., Trin. 830).

['Así me gusta, te felicito. Tú sabes tratar a los hombres según su rango, como se debe.']

Sin embargo, esta no es indispensable, como puede verse en (34), ejemplo que el hablante se dirige a sí mismo y sobre cuyo valor no hay duda - gracias además al comentario de Donato (35) -, y en (36), que da cuenta de la pervivencia de este valor en época clásica:

(34) SY. Edepol, Syrisce, te curasti molliter

lauteque munus administrasti tuom:

abi. [...] (Ter., Ad. 763-765).

['iPor Pólux, Sirito, qué regaladamente te has tratado y qué primorosamente has cumplido con tu obligación! iBravo!']

(35) ABI uerbum uel sibi uel alteri cum laudatione blandientis. Nam sic dicunt, qui iam compotes sunt uotorum omnium perfectique in rebus uniuersis (Don., ad Ter., Ad. 765).

['ABI es una palabra de halago con felicitación bien a sí mismo bien a otro. Pues así dicen quienes han visto cumplidos todos sus deseos y han llevado a término todas sus asuntos.']

(36) non es auarus, abi (Hor., Epist. 2, 2, 205).

['No eres avaro. Estupendo.']

Según la propuesta de Löfstedt (1966: p. 97), el origen de estos usos se encontraría en un desplazamiento metafórico basado en el sentido directivo originario de esta unidad, que sería pronunciada por alguien que ha sometido a su interlocutor a una prueba de la que este último ha salido airoso. Sin embargo, si bien este valor podría defenderse en aquellos empleos en los que el locutor que se sirve de abi ocupa una posición jerárquica de superioridad con respecto al oyente, como es el caso de (31) y de (32), ${ }^{31}$ esa primacía

30 Como ocurre en parte en este ejemplo, $a b i$ asume un valor claramente irónico en su primer uso en el siguiente fragmento: PA. Sati' iam dictum habeo. SO. Sed quid tu? Confitere ut te autumo? / PA. Fatear, si ita sim. SO. Iam abi, uicisti. PA. Abi nunc iam ergo. SO. Hoc mi expedi, / quo agis? [...] (Plaut., Persa 214-216).

31 En ambos casos se ha producido una inversión de la jerarquía habitual amo-esclavo, consistente en que, dada la necesidad que tiene el primero de la ayuda del segundo en asuntos amorosos, es el esclavo, verdadero protagonista de las comedias, el que toma las riendas de la situación y ocupa la posición preminente. 
del hablante desaparece en (33) inserto en una plegaria gratulatoria al dios Neptuno tras el regreso de un viaje por mar, y es irrelevante en (34), donde el hablante pronuncia abi en el marco de un monólogo y referido a sí mismo.

Parece, por tanto, conveniente matizar esta interpretación y considerar que, partiendo de su valor genérico de iniciación de cierre, en el que, como se ha dicho, expresa sencillamente el cumplimiento de los objetivos comunicativos, abi habría asumido un valor pragmático secundario, es decir, se habría repragmaticalizado, para expresar la felicitación, en consonancia con la idea conversacional del cumplimiento de un objetivo, que deja de ser meramente comunicativo para referirse a una actitud o un logro. De este modo, a partir de la idea de «ya he conseguido lo que quería de este intercambio, puedes marcharte» pasamos a otra más compleja del tipo «he conseguido lo que quería de mi trato contigo, lo has hecho bien (= te felicito)», cuya fijación se observa en los casos en que abi no aparece acompañado de la especificación léxica de la fuerza ilocutiva del mensaje - como en (34) -.

Con este valor, al igual que en el caso de los reproches, nos encontramos ya en el ámbito de la plena subjetividad del hablante, que se corresponde con la tercera tendencia propuesta por Trauggot («Meanings tend to become increasingly based in the speaker's subjective belief state/attitude toward the proposition» [Traugott 1989: pp. 34-35]). Reproches y felicitaciones constituyen así dos facetas del mismo fenómeno: la subjetivación de la valoración del éxito o fracaso de los objetivos propuestos para un intercambio entre dos individuos.

En el caso de las felicitaciones encontramos además una evidencia adicional que nos permite defender la subjetivación de $a b i$. Uno de los tres mecanismos que posee el latín arcaico para la manifestación de este acto de habla es la expresión de la alegría o la sorpresa (Unceta Gómez 2016b: pp. 280-282), nociones frecuentemente expresadas en el registro oral y coloquial por medio de interjecciones. Así, tanto eug(a)e! y eugepae!, préstamos griegos que expresan una alegría enérgica (Unceta Gómez 2012: pp. 376-378), como $e u$ !, cuyo valor básico de sorpresa se solapa (especialmente en la obra de Terencio) con el adverbio griego $\varepsilon \tilde{u}$ ('bien') (Unceta Gómez 2012: pp. 369-371), pueden desarrollar una función ilocutiva subsidiaria de felicitación, como se aprecia en los siguientes ejemplos: (37) [...] apud «te eos hic deuortier

dicam hospitio. PE. Eugae eugae, lepide, laudo commentum tuom! (Plaut., Mil. 240-241). ['Diré que los amantes se alojan como huéspedes en tu casa. PERIPLECTÓMENO. iBravo! iBravo! iMaravilloso! iMagnifica ocurrencia!']

(38) [...] GET. Eu noster, recte. [...] (Ter., Phorm. 398).

['Bien por mi amo! iBravo!']

Tomando como modelo ejemplos como los de (37) y (38), podemos asumir que abi ha desarrollado en sus usos como felicitación un significado mirativo o de alegría que lo asemeja a las mencionadas interjecciones primarias. Si bien se nos escapan los factores prosódicos, indispensables para su correcta interpretación, únicamente en estos casos sería posible considerar abi como una interjección impropia. ${ }^{32}$

32 Este valor mirativo se reconoce también en algunos usos de otras formas verbales gramaticalizadas como em (similar a español itoma!; cf. Unceta Gómez 2017), o quaeso y obsecro ('por favor'; Unceta Gómez 2015). 


\section{Conclusiones}

Hasta aquí se han identificado los valores pragmáticos desarrollados por el imperativo del verbo de movimiento abire, que pueden considerarse ya muy mecanizados en el latín arcaico de las comedias. La Tabla 2 presenta la distribución de esos valores. ${ }^{33}$

\begin{tabular}{|l|c|c|}
\hline \multicolumn{1}{|c|}{ Valor } & Ejemplos & Porcentaje \\
\hline Sentido etimológico & 92 & $44 \%$ \\
\hline Imperativo asociativo & 42 & $20 \%$ \\
\hline Refuerzo AH directivo & 5 & $2 \%$ \\
\hline Cierre de diálogo & 34 & $16 \%$ \\
\hline Desacuerdo/Reproche & 27 & $13 \%$ \\
\hline Acuerdo/Felicitación & 7 & $3 \%$ \\
\hline TOTAL & 207 & $100 \%$ \\
\hline
\end{tabular}

Tabla 2. Distribución de los valores de abi.

El recorrido de estas evoluciones puede plantearse como sigue: a partir de un valor directivo básico, que indica un movimiento espacial, se desarrollan los llamados usos asociativos, sintagmas con un nivel de gramaticalización elevado, que darán lugar a la función de pura incitación a la acción, es decir, de intensificación del acto de habla directivo. Por otra parte, vinculado al valor del verbo abire en el lenguaje técnico teatral para la expresión de la salida de escena, abi desarrollará un valor iniciativo de cierre del intercambio comunicativo. En esa función, a través de su subjetivación, abi asumirá valores expresivos adicionales: el reproche y la aprobación o felicitación, donde esta unidad asume un estatuto cercano a lo interjectivo.

El desarrollo como marcador pragmático de desacuerdo o reproche podría, con todo, recibir una interpretación diferente. Daniels (2014: p. 231) propone que el marcador español venga desarrolló su valor de desacuerdo, usado de manera reactiva, a través de su participación en construcciones hortativas prohibitivas: «The discourse marker of disagreement arose in the late twentieth century, from the prohibitive use of venga. This reanalysis can be attributed to metaphorical (or metonymic) extension of conventionalized conversational implicatures: (1) The speaker did not want the addressee to do X, and/or (2) the speaker wanted the addressee to stop doing X, and/or (3) the speaker did not agree with what the addressee was doing. This third implicature was carried over to the discourse marker of disagreement by way of metaphorical extension. Thus, the speaker used venga ( $y a$ ) to express disagreement with the thoughts of the addressee rather than the actions of the addressee [...]. With this metaphorical extension from action to thought complete, venga (ya) gained greater syntactic freedom and was used in syntactic environments separate from imperatives and prohibitives» (Daniels 2014: p. 241).

33 Los porcentajes que se presentan en esta tabla son meras aproximaciones, pues en ocasiones es perceptible más de un valor, dada la polifuncionalidad inherente a este tipo de desarrollos. 
En el caso de $a b i$, sin embargo, no parece posible defender este paso, pues, salvo en Plaut., Persa 490 (abi, ne iura, sati' credo), no se documenta su combinación con construcciones prohibitivas, de modo que resulta más adecuado pensar en el desplazamiento metafórico mencionado en $\$ 3.3$.

En todo caso, cabe señalar que la documentación simultánea de todas estas funciones impide hacer cualquier afirmación tajante sobre el sentido de estas evoluciones, puesto que debemos abordar una propuesta diacrónica sobre un corte sincrónico en el que ya se documentan todos los valores de esta unidad. Sin embargo, aunque en latín no hay más muestras de este tipo de evolución en verbos de movimiento (por ejemplo, ni uade ni $i$, que también dan lugar a construcciones asociativas, desarrollan estos valores), otras lenguas europeas proporcionan ejemplos paralelos con gran rendimiento pragmático, como la partícula va del francés, la italiana va là, las españolas anda y venga, o la alemana geh (weiter), ${ }^{34}$ unidades para las que se proponen evoluciones semejantes $\mathrm{y}$, como se ha señalado, apuntalan algunos de los desarrollos propuestos.

A la vista del análisis ofrecido en este trabajo y de la explicación sobre las vías de pragmaticalización de $a b i$, parece clara, por último, la necesidad de repensar las funciones pragmáticas de ciertas unidades, consideradas como parte del paradigma de los lexemas de que derivan o, a lo sumo, como interjecciones, y ofrecer un nuevo análisis lexicográfico de las mismas, reelaborando y añadiendo entradas en los diccionarios de referencia. Los avances en el análisis pragmático de lenguas modernas que se están llevando a cabo en los últimos años aportan unos métodos que pueden proporcionar una mejora sustancial de nuestro conocimiento de las lenguas antiguas.

\section{Bibliografía}

Barbini, M. A. (1966). Interferenze fra imperativi ed interiezioni. Giornale Italiano di Filologia, 19, 357-363.

Blas Arroyo, J. L. (1998). Un caso de variación pragmática: sobre la ampliación significativa de un marcador discursivo en el español actual. Aspectos estructurales y sociolingüísticos. Analecta Malacitana, 21, 543-572.

Briz, A., Pons, S., \& Portolés, J. (Coords.). (2008). Diccionario de partículas discursivas del español. [Disponible online en www.dpde.es].

Chahoud, A. (2010). Idiom(s) and literariness in classical literary criticism. In E. Dickey, \& A. Chahoud (Eds.), Colloquial and literary Latin (pp. 42-63). Cambridge: Cambridge University Press.

Company Company, C. (2004). Gramaticalización por subjetivización como prescindibilidad de la sintaxis. Nueva Revista de Filología Hispánica, 52(1), 1-27.

Daniels, K. (2014). On the grammaticalization of venga 'come' as a discourse marker in Peninsular Spanish. In M. Devos, \& J. van der Wal (Eds.), COME and GO off the beaten grammaticalization path (pp. 219-247). Berlin - Boston: Walter de Gruyter.

Dickey, E. (2006). The use of latin sis as a focus-marking clitic particle. Oxford University Working Papers in Linguistics, Philology $\mathcal{E}$ Phonetics, 11, 21-25.

34 Paralelos señalados por Hofmann (32003: p. 151) y Löfstedt (1966: p. 97). 
Diewald, G. (2011). Pragmaticalization (defined) as grammaticalization of discourse functions. Linguistics, 49(2), 365-390.

Dostie, G. (2004). Pragmaticalisation et marqueurs discursifs: analyse sémantique et traitement lexicographique. Bruxelles: De Boeck.

García Hernández, B. (2015). El grupo preverbial del lat. ire ('ir’). Funciones sémicas y clasemáticas. In P. Anreiter et al. (Eds.), Festschrift für Manfred Kienpointner zum 60. Geburtstag (pp. 111-123). Wien: Praesens-Verlag.

Ghezzi, C. (2014). The development of discourse and pragmatic markers. In C. Ghezzi, \& C. Molinelli (Eds.), Discourse and pragmatic markers form Latin to the Romance languages (pp. 10-26). Oxford: Oxford University Press.

González Vázquez, C. (2014). Diccionario Akal del Teatro latino. Léxico, dramaturgia, escenografía (Nueva edición revisada y ampliada). Madrid: Akal.

Hofmann, J. B. (2003). La lingua d'uso latina (Introduzione, traduzione e note a cura di L. Ricottilli; 3. ed.). Bolonia: Pàtron. [= Lateinische Umgangssprache. Heidelberg: Winter 1951].

Hough, J. N. (1945). The numquid uis formula in Roman Comedy. American Journal of Philology, 66, 282-302.

Jacobs, A., \& Jucker, A. H. (1995). The historical perspective in pragmatics. In A. H. Jucker (Ed.), Historical Pragmatics (pp. 3-33). Amsterdam - Philadelphia: John Benjamin.

Kauer, R., \& Lindsay, W. M. (Eds.). (1926). P. Terenti Afri Comoediae. Oxonii: E Typographeo Clarendoniano.

Lindsay, W. M. (Ed.). (1903-1904). T. Macci Plauti Comoediae. Oxonii: E Typographeo Clarendoniano.

Löfstedt, L. (1966). Les expressions du commandement et de la défense en Latin et leur survie dans les langues romaines. Helsinki: Société Néophilologique.

Mauri, C., \& Sansò, A. (2014). Go and come as sources of directive constructions. In M. Devos, \& J. van der Wal (Eds.), COME and GO off the beaten grammaticalization path (pp. 165-184). Berlin - Boston: Walter de Gruyter.

Pinkster, H. (2015). The Oxford Latin Syntax, I: The Simple Clause. Oxford: Oxford University Press.

Risselada, R. (1993). Imperatives and other directive expressions in Latin. A study in the pragmatics of a dead language. Amsterdam: J. C. Gieben.

Risselada, R. (1994). Modo and sane, or what to do with particles in Latin directives. In J. Herman (Ed.), Linguistic studies on Latin. Selected papers from the $6^{\text {th }}$ International Colloquium on Latin Linguistics (pp. 319-343). Amsterdam - Philadelphia: John Benjamins.

Roesch, S. (2002). Les stratégies de clôture du dialogue dans les comédies de Plaute. In A. Bolkestein et al. (Eds.), Theory and description in Latin linguistics (pp. 317-332). Amsterdam: Brill.

Román Bravo, J. (Transl.). (2012). Comedia latina. Obras completas de Plauto y Terencio (Edición, introducciones y notas de Rosario López Gregoris; Bibliotheca aurea). Madrid: Cátedra.

Rubio Fernández, L. (Transl.). (1978). Apuleyo: El asno de oro. Madrid: Gredos.

Torrego, M. E. (2009). Ablativo. In J. M. Baños, Sintaxis del latín clásico (pp. 211-249). Madrid: Liceus.

Traugott, E. C. (1989). On the rise of epistemic meanings in English: An example of subjectification in semantic change. Language, 65, 31-55.

Traugott, E. C. (1995). Subjectification in grammaticalization. In D. Stein, \& S. Wright (Eds.), Subjectivity and subjectivisation. Linguistic perspectives (pp. 31-54). Cambridge: Cambridge University Press. 
Unceta Gómez, L. (2009). La petición verbal en latín: estudio léxico, semántico y pragmático. Madrid: Ediciones Clásicas.

Unceta Gómez, L. (2012). Cuando los sentimientos irrumpen: Valores expresivos de las interjecciones primarias en las comedias de Plauto. In R. López Gregoris (Ed.), Estudios sobre teatro romano: El mundo de los sentimientos y su expresión (pp. 347-395). Zaragoza: Pórtico.

Unceta Gómez, L. (2014). La politesse linguistique en latin: Bilan d'une étude en cours. In Dictionnaire Historique et Encyclopédie Linguistique du Latin, Paris-Sorbonne. [Disponible online en: www. linglat.paris-sorbonne.fr/encyclopedie_linguistique:notions_linguistiques:syntaxe:formules_ de_politesse].

Unceta Gómez, L. (2015). Sobre el proceso de subjetivación de algunas formas verbales en la lengua de la comedia romana. In G. Haverling (Ed.), Latin Linguistics in the Early 21st Century. Acts of the 16th International Colloquium on Latin Linguistics, Uppsala, June 6th-11th, 2011 (pp. 468-479). Uppsala: Uppsala Universitet.

Unceta Gómez, L. (2016a). Cuando los sentimientos irrumpen: Análisis de las interjecciones secundarias en las comedias de Plauto. In B. García-Hernández, \& A. Penas Ibáñez (Eds.), Semántica latina y románica. Unidades de significado conceptual y procedimental (pp. 213-241). Bern: Peter Lang.

Unceta Gómez, L. (2016b). Congratulations in Latin Comedy: Types and functions. Journal of Politeness Research, 12(2), 267-290.

Unceta Gómez, L. (2017). Grammaticalization, pragmaticalization... interjectionalization? The case of Latin em. In C. Denizot, \& O. Spevak (Eds.), Pragmatic approaches to Latin and Ancient Greek. Amsterdam - Philadelphia: John Benjamins (en prensa).

Prof. Dr. Luis Unceta Gómez / luis.unceta@uam.es

Departamento de Filología Clásica

Universidad Autónoma de Madrid, Facultad de Filosofía y Letras

Ciudad Universitaria de Cantoblanco, 28049 Madrid, España 